\title{
Long non-coding RNA MIR22HG promotes osteogenic differentiation of bone marrow mesenchymal stem cells via PTEN/ AKT pathway
}

\author{
Chanyuan Jin', Lingfei Jia ${ }^{2,3}$, Zhihui Tang ${ }^{1}$ and Yunfei Zheng ${ }^{4}$
}

\begin{abstract}
Osteoporosis is a prevalent metabolic bone disease characterized by low bone mineral density and degenerative disorders of bone tissues. Previous studies showed the abnormal osteogenic differentiation of endogenous bone marrow mesenchymal stem cells (BMSCs) contributes to the development of osteoporosis. However, the underlying mechanisms by which BMSCs undergo osteogenic differentiation remain largely unexplored. Recently, long noncoding RNAs have been discovered to play important roles in regulating BMSC osteogenesis. In this study, we first showed MIR22HG, which has been demonstrated to be involved in the progression of several cancer types, played an important role in regulating BMSC osteogenesis. We found the expression of MIR22HG was significantly decreased in mouse BMSCs from the osteoporotic mice and it was upregulated during the osteogenic differentiation of human BMSCs. Overexpression of MIR22HG in human BMSCs enhanced osteogenic differentiation, whereas MIR22HG knockdown inhibited osteogenic differentiation both in vitro and in vivo. Mechanistically, MIR22HG promoted osteogenic differentiation by downregulating phosphatase and tensin homolog (PTEN) and therefore activating AKT signaling. Moreover, we found MIR22HG overexpression promoted osteoclastogenesis of RAW264.7 cells, which indicated that MIR22HG played a significant role in bone metabolism and could be a therapeutic target for osteoporosis and other bone-related diseases.
\end{abstract}

\section{Introduction}

Osteoporosis is one of the most common metabolic bone diseases which results from the disrupted balance between bone formation and resorption ${ }^{1}$. With the progressive aging of the global population, the incidence of osteoporosis is increasing dramatically which leads to an increased risk of fractures and exerts a strong impact on morbidity, and even mortality ${ }^{2,3}$. However, the pathological mechanisms of osteoporosis have not been fully understood yet. BMSCs are precursors that can

\footnotetext{
Correspondence: Zhihui Tang (tang_zhihui@live.cn) or

Yunfei Zheng (yunfei_zheng@bjmu.edu.cn)

${ }^{1}$ The Second Clinical Division of Peking University School and Hospital of Stomatology, 100081 Beijing, China

${ }^{2}$ Central Laboratory, Peking University School and Hospital of Stomatology, 100081 Beijing, China

Full list of author information is available at the end of the article

Edited by A. Stephanou
}

differentiate into osteoblasts and adipocytes in bone and play an important role in maintaining bone homeostasis. Previous studies showed the impaired osteogenic differentiation capacity of BMSCs contributes to the progression of osteoporosis ${ }^{4}$. However, the molecular basis involved in the osteogenic differentiation of BMSCs remains unclear. Therefore, a deeper insight into the mechanisms of BMSC osteogenesis will provide new strategies for treating and preventing osteoporosis.

Long non-coding RNAs (lncRNAs) are a class of nonprotein-coding RNA transcripts with lengths longer than 200 nucleotides. Growing amounts of evidences have shown that lncRNAs are important regulators in diverse biological processes and diseases including osteoporosis ${ }^{5-}$ 7. A good number of lncRNAs have been demonstrated to play critical roles in the osteogenic differentiation of BMSCs $^{8-10}$. For example, lncRNA MALAT1 was 
downregulated in BMSCs from osteoporosis rats compared with the normal rats, and could inhibit osteogenesis through MAPK signaling ${ }^{11}$.

LncRNA MIR22HG, which is located on chromosome $17 \mathrm{p} 13.3$, has been discovered to play roles in a variety of cancers. Many previous studies revealed that MIR22HG exhibited tumor-suppressive role in several types of cancer including lung cancer, hepatocellular carcinoma, endometrial cancer, gastric cancer and cholangiocarcinoma, and its low expression was associated with poor prognosis $^{12-17}$. Conversely, some studies showed that MIR22HG played an oncogenic function in several cancer types such as glioblastoma, and MIR22HG knockdown inhibits the invasion and proliferation of cancer cells ${ }^{18}$. However, no study reported the function of MIR22HG in the regulation of bone metabolism.

In this study, we established osteoporosis mice model and found MIR22HG was significantly downregulated in mouse BMSCs (mBMSCs) from osteoporosis mice compared with the normal mice. MIR22HG was increased during the osteogenic differentiation of human BMSCs (hBMSCs) and acted as a positive regulator for hBMSC osteogenesis not only in vitro, but also in vivo. Mechanistically, we demonstrated that MIR22HG promoted osteogenic differentiation of hBMSCs via PTEN/AKT signaling. Overall, these findings suggested that MIR $22 H G$ might serve as a promising therapeutic target for osteoporosis treatment and prevention.

\section{Materials and methods \\ Cell cultivation}

Primary hBMSCs, human adipose-derived stem cells (hASCs) and RAW264.7 cells were purchased from ScienCell company (Carlsbad, CA, USA). Cells were cultured in proliferation medium (PM) consisting of DMEM supplemented with $10 \%$ fetal bovine serum and $1 \%$ antibiotics. For osteogenic differentiation, hBMSCs and hASCs were induced in osteogenic media (OM) composed of standard PM supplemented with $100 \mathrm{nM}$ dexamethasone, $0.2 \mathrm{mM}$ ascorbic acid, and $10 \mathrm{mM}$ $\beta$-glycerophosphate. All cell-based in vitro experiments were performed at least three times.

\section{Transfection}

The recombinant lentiviruses containing full-length MIR22HG and the scramble control (NC) were purchased from Cyagen Biosciences (Guangzhou, China). Recombinant lentiviruses targeting MIR22HG (shMIR22HG-1 and shMIR22HG-2) and the nontargeting scramble control (shNC) were obtained from GenePharma Co. (Shanghai, China). The plasmid pcDNA3.1(+)-PTEN and pcDNA3.1(+), small interfering RNAs targeting PTEN (si-PTEN) and the scramble control (si-NC) were obtained from Integrated Biotech
Solutions Co. (Shanghai, China). The sequences are listed in Supplemental Table 1.

\section{Alkaline phosphatase (ALP) staining and activity}

ALP staining and ALP activity assay were performed as described previously ${ }^{19} .7$ days after osteogenic induction, cells were rinsed with PBS, fixed with $4 \%$ paraformaldehyde, incubated with the NBT/BCIP staining kit (CoWin Biotech, Beijing, China). ALP activity was assayed with the ALP Activity Kit (Biovision, Milpitas, CA) and normalized to the total protein contents.

\section{Alizarin red $\mathrm{S}$ staining and quantification}

14 days after osteogenic induction, cells were washed with PBS, fixed with $4 \%$ paraformaldehyde, and stained with $1 \%$ Alizarin red S solution for $20 \mathrm{~min}$. To quantify the degree of mineralization of hBMSCs, the stain was solubilized by cetylpyridinium chloride and quantified by a spectrophotometer at $570 \mathrm{~nm}$.

\section{RNA isolation and quantitative reverse transcription- polymerase chain reaction (qRT-PCR) analysis}

Total RNA was extracted from cells with TRIzol Reagent (Invitrogen, Carlsbad, CA, USA). Then the RNA was reverse transcribed into cDNA using PrimeScript RT Reagent Kit (Takara, Tokyo, Japan). qRT-PCR was amplified with SYBR Green Master Mix (Roche Applied Science, Mannheim, Germany) on a 7500 Real-Time PCR Detection system (Applied Biosystems, Foster City, CA, USA). The comparative cycle threshold (CT) method was used to calculate the relative gene expression. The primers used for Bulge-loop reverse-transcription PCR for microRNA-22 (miR-22) and U6 were purchased from RiboBio (Guangzhou, China). The sequences of primers used are listed in Supplemental Table 1.

\section{Western blot analysis}

Cells were lysed using radioimmunoprecipitation assay (RIPA) lysis buffer. Samples were separated by electrophoresis and then transferred to PVDF membranes (Millipore, Billerica, MA, USA). The membranes were blocked with skimmed milk and incubated with primary antibodies against AKT (Cell Signaling Technology, Beverly, MA, USA), phosphorylated-AKT (Ser473) (Cell Signaling Technology), PTEN (Cell Signaling Technology) and GAPDH (HuaxingBio Science, Beijing, China) at $4{ }^{\circ} \mathrm{C}$ overnight. Then, the membranes were incubated with secondary antibodies for $1 \mathrm{~h}$ at room temperature. Signals were visualized using the ECL Kit (CoWin Biotech).

\section{Establishment of ovariectomized mouse model}

All animal experiments procedures were performed in compliance with the technical guidelines and approved by the Peking University Animal Care and Use Committee. 
Twenty BALB/c female mice (8 weeks, $18-20 \mathrm{~g}$ ) were purchased from Vital Co. (Beijing, China) and randomly divided to two groups: the ovariectomized (OVX) group, non-OVX control (SHAM) group. The mice in the OVX group received bilateral ovariectomy surgery. As a control, the mice in the SHAM group were subjected to removal of adipose tissue near the ovaries. After 6 weeks, mice were sacrificed under anesthesia and the femurs or tibias were collected for the related assays.

mBMSCs were isolated and purified from the tibias of SHAM and OVX mice as described previously ${ }^{20}$. Flow cytometry analysis sorting was performed to screen mBMSCs which were positive for CD44 and CD29 but negative for $\mathrm{CD} 45$. The multilineage differentiation potential of isolated mBMSCs was evaluated via osteogenic differentiation and adipogenic differentiation. For osteogenic differentiation, mBMSCs were induced in OM for 7 days and ALP staining was performed. For adipogenic differentiation, mBMSCs were cultured in standard PM supplemented with $50 \mathrm{nM}$ insulin, $100 \mathrm{nM}$ dexamethasone, $0.5 \mathrm{mM}$ 3- isobutyl-1-methylxanthine, and $200 \mathrm{mM}$ indomethacin for 14 days. Oil red $\mathrm{O}$ staining was performed as described before ${ }^{21}$.

\section{Micro CT analyses of mice}

Six weeks after the ovariectomy surgery, femurs were harvested and scanned using a Micro CT system (pixel size $8.82 \mathrm{~mm}$, working voltage $80 \mathrm{kV}$, working current $500 \mathrm{~mA}$, exposure time $1500 \mathrm{~ms}$ ). Three-dimensional reconstruction was performed with multimodal 3D visualization software (Inveon, Siemens, Munich, Germany). Bone volume/total volume (BV/TV), trabecular number (Tb.N), and trabecular spacing (Tb.Sp) in the trabecular region $(1.5 \mathrm{~mm}$ distal to the proximal epiphysis) were analyzed using an Inveon Research Workplace (Siemens). Then the femurs were decalcified in 10\% ethylene diamine tetraacetic acid (EDTA) and stained with hematoxylin and eosin (HE).

\section{Ectopic bone formation in vivo}

The hBMSCs infected with lentivirus were induced in OM for 7 days before the in vivo experiments. Next, $5 \times$ $10^{6}$ cells were mixed with $7 \mathrm{~mm} \times 5 \mathrm{~mm} \times 2 \mathrm{~mm}$ Bio-Oss Collagen (Geistlich, GEWO GmbH, Baden-Baden, Germany), incubated for $1 \mathrm{~h}$ at $37^{\circ} \mathrm{C}$, and then implanted subcutaneously into the dorsal surface of 8 -week-old BALB/c homozygous nude $(\mathrm{nu} / \mathrm{nu})$ female mice $(n=6$ per group). The mice were randomized into 5 groups: shNC-hBMSCs/Collagen group, shMIR22HG-1hBMSCs/Collagen group, shMIR22HG-2-hBMSCs/Collagen group, NC-hBMSCs/Collagen group and MIR22HG-hBMSCs/Collagen group. The implants were collected after 8 weeks and fixed with $4 \%$ formalin, decalcified, and embedded in paraffin wax. Sections $(5 \mu \mathrm{m})$ were cut and stained with HE, Masson's trichrome and immunohistochemical analysis. Immunohistochemical analysis was performed to detect the expression level of osteocalcin (OCN) (anti-OCN, Cell Signaling Technology).

\section{RNA sequencing}

Total RNA isolated from MIR22HG knockdown or shNC hBMSCs was used as input material for the RNA sample preparations. cDNA was synthesized and then PCR was performed with phusion high-fidelity DNA polymerase, universal PCR primers and index primer. Finally, PCR products were purified (AMPure XP system) and library quality was evaluated on the Agilent Bioanalyzer 2100 system. Thereafter, the clustering of the indexcoded samples was performed on a cBot Cluster Generation System using TruSeq PE Cluster Kit v3-cBot-HS (Illumia). After cluster generation, the library preparations were sequenced on an Illumina NovaSeq platform and $150 \mathrm{bp}$ paired-end reads were generated. Differentially expressed genes were defined as those with a $P$ value $<$ 0.05 and a Fold change $>2$.

\section{Fluorescent in situ hybridization (FISH)}

FISH was conducted with a Fluorescent In Situ Hybridization Kit (RiboBio). Briefly, cells were washed in PBS, fixed with $4 \%$ formaldehyde, and then permeabilized in PBS containing $0.5 \%$ Triton $\mathrm{X}-100$ at $4{ }^{\circ} \mathrm{C}$ for $5 \mathrm{~min}$. Subsequently, cells were prehybridizated at $37^{\circ} \mathrm{C}$ for $30 \mathrm{~min}$ and then hybridized with anti-MIR22HG, anti-U6, or anti-18S oligodeoxynucleotide probe at $37^{\circ} \mathrm{C}$ overnight. Then the cells were stained with DAPI for detection of nuclei and captured using a confocal microscope (Carl Zeiss, Germany).

\section{Subcellular fractionation}

Nuclear and cytoplasmic fractions of hBMSCs were separated using a Nuclei Isolation Kit (Invent-biotech, Minnesota, USA). RNA was isolated from both fractions with Trizol and then reverse transcribed into cDNA. The RNA expression was quantified by qRT-PCR as described above $^{19}$. MALAT1 and GAPDH were used as fractionation indicators. The primers used are listed in Supplementary Table 1.

\section{Colocalization of MIR22HG and PTEN}

The colocalization of MIR22HG and PTEN was performed as previously described ${ }^{22}$. After hybridization with an anti-MIR22HG probe (RiboBio), hBMSCs were washed with PBS and incubated with the PTEN antibody at $4{ }^{\circ} \mathrm{C}$ overnight. The next day, the cells were incubated in the dark using a Dylight 488-conjugated secondary antibody (Abbkine, California, USA) and stained with DAPI. 
Tartrate resistant acid phosphatase (TRAP) staining

For osteoclast differentiation, RAW264.7 cells were treated with murine receptor activator of nuclear factorkB ligand (RANKL) (50 ng/ml, Peprotech, NJ, USA) for 5 days. TRAP staining was performed using an acid phosphatase kit (Sigma-Aldrich, St. Louis, MO, USA). Images of TRAP-positive multinucleated cells (containing $\geq 3$ nuclei/cell) were recorded with a microscope.

\section{Statistical analysis}

All statistical values were calculated using SPSS version 16.0 (SPSS Inc., Chicago, IL, USA). Independent sample $t$ test was used to evaluate statistical differences between two groups, and one-way analysis of variance (ANOVA) was used for data of multiple groups. Results were expressed as mean \pm standard deviation. A $p$ value less than 0.05 was considered statistically significant.

\section{Results}

MIR22HG was decreased in OVX mice

Recent studies indicated that osteoporosis may be caused by abnormal osteogenic differentiation of BMSCs. To investigate the pathological mechanisms of osteoporosis, we constructed osteoporotic model by ovariectomy. HE staining, Micro CT images and analyses showed that the bone trabeculae of OVX mice was remarkably decreased compared with SHAM mice (Fig. $1 \mathrm{a}-\mathrm{d})$. At the same time, mBMSCs from both SHAM and OVX mice were isolated, sorted by flow cytometry and determined with differentiation assays (Supplementary Fig. 1A-C). Based on our previous work, expression levels of several interesting genes were chosen to be measured by qRT-PCR and we found many genes were differentially expressed after ovariectomy. Among them, MIR22HG appeared to be one of the most differentially expressed genes. As shown in Fig. 1f, the MIR22HG expression level was significantly decreased in OVX mice, meanwhile the expression level of $R U N X 2$, an osteogenic marker, was also significantly decreased in mBMSCs from OVX mice (Fig. 1e). These results suggested the potential involvement of MIR22HG in the compromised osteogenesis of osteoporotic BMSCs.

\section{MIR22HG was increased during osteogenesis of hBMSCs}

To elucidate whether MIR22HG plays a role in osteogenic differentiation of BMSCs, we first attempted to profile MIR22HG expression pattern in hBMSCs after osteogenic induction. qRT-PCR result indicated that the expression of $M I R 22 H G$ was significantly upregulated (Fig. 2a). As expected, the mRNA levels of osteogenesisrelated genes $R U N X 2, A L P$, and $O C N$ were significantly increased (Fig. 2b-d).

\section{MIR22HG promoted osteogenic differentiation}

To evaluate the function of MIR22HG in the osteogenic differentiation of hBMSCs, we used lentivirus to knockdown or overexpress MIR22HG in hBMSCs (Supplementary Fig. 2A-C). Seven days after culturing the transfected cells in PM or OM, ALP activity was significantly increased in the MIR22HG overexpression group but decreased in the MIR22HG knockdown group (Fig. 3a, b). Consistently, the mineralized nodules, as detected by ARS staining and quantification on day 14, was increased in the MIR22HG overexpression group but reduced in the MIR22HG knockdown group (Fig. 3c, d). Moreover, the mRNA levels of RUNX2, ALP, and OCN were decreased in the MIR22HG knockdown group but upregulated in $M I R 22 H G$ overexpression group (Fig. 3e-g).

Additionally, we also investigated the effect of MIR22HG on osteogenesis of hASCs. Results showed MIR22HG played a similar function in hASCs (Supplementary Fig. 3A-F). All of these results revealed a positive role of $M I R 22 H G$ in regulating osteogenesis.

\section{MIR22HG promoted bone formation of hBMSCs in vivo}

To further discover the role of $M I R 22 H G$ in osteogenesis, we next tested whether MIR22HG could regulate bone formation potential of hBMSCs in vivo. HE staining and Masson's trichrome staining showed MIR22HG overexpression group formed more bone-like tissues compared with its control group (NC), whereas MIR22HG knockdown dramatically inhibited the efficiency of new bone formation compared with the control group (shNC). Moreover, we found the expression level of OCN was higher in MIR22HG overexpression group but lower in MIR22HG knockdown group as revealed by immunohistochemical staining (Fig. 4).

\section{Differentially expressed genes in MIR22HG knockdown BMSCs}

To further investigate the underlying mechanisms of $M I R 22 H G$ in the regulation of hBMSC osteogenesis, shMIR22HG-1, shMIR22HG-2, and their negative control (shNC) hBMSCs were collected and subjected to RNA sequencing. Heat map revealed the genes that were differentially expressed following MIR22HG knockdown (Fig. 5a). Among these genes, 278 genes were upregulated and 112 genes were downregulated (Fig. 5b). Furthermore, KEGG pathway analysis was applied to identify the pathways that were differentially expressed by $M I R 22 H G$ knockdown. Among the downregulated signaling pathways, phosphatidylinositol 3-kinase (PI3K)/AKT signaling which is closely associated with osteogenic differentiation appeared to be one of the most enriched pathways (Fig. 5c). 


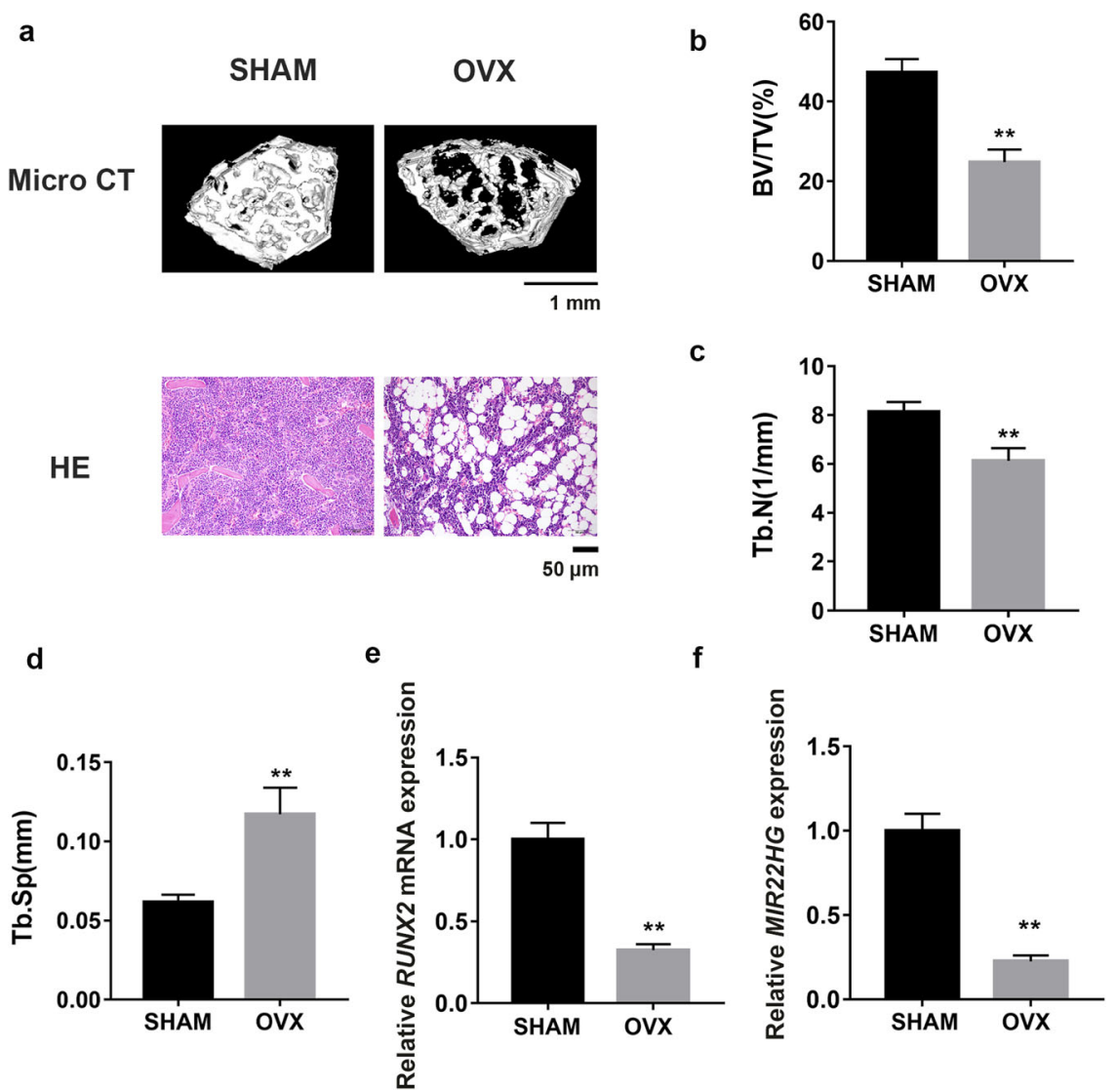

Fig. 1 MIR22HG expression was decreased in OVX mice. a Representative images of Micro CT and HE staining from SHAM and OVX mice indicated the bone loss of OVX mice compared to SHAM mice. Scale bars for Micro CT and HE staining represent $1 \mathrm{~mm}$ and $50 \mu \mathrm{m}$, respectively. b-d Trabecular bone volume/tissue volume (BV/TV), trabecular number (Tb.N), and trabecular spacing (Tb.Sp) were detected in SHAM and OVX mice. e, $\mathbf{f}$ Expression levels of RUNX2 and MIR22HG tested in mBMSCs from OVX mice in contrast to SHAM mice, determined by qRT-qPCR. All data are shown as mean $\pm S D$, ${ }^{* *} P<0.01$, compared with SHAM group.

Furthermore, Western blot showed the level of p-AKT was significantly decreased in the shMIR22HG hBMSCs (Fig. 6a, b), which was consistent with the result of RNA sequencing. We therefore hypothesized that MIR22HG might promote the osteogenic differentiation of hBMSCs with the involvement of AKT signaling.

\section{MIR22HG knockdown impaired osteogenesis via inhibiting AKT activation}

To determine whether MIR22HG knockdown inhibits hBMSC osteogenesis through inhibiting AKT signaling, we treated MIR22HG knockdown hBMSCs and the negative control group (shNC) with AKT signaling activator 740 Y-P. Western blot showed 740 Y-P significantly upregulated the level of p-AKT (Supplementary Fig. 4A, B). We next examined whether the addition of 740 Y-P could rescue the impaired osteogenesis caused by MIR22HG knockdown. ALP staining and activity showed the administration of 740 Y-P counteracted the impairments of MIR22HG knockdown on ALP activity (Fig. 6c, d). Similarly, ARS staining and quantification showed 740 Y-P treatment partially abrogated the inhibitory effect of MIR22HG knockdown on mineralization (Fig. 6e, f). Moreover, qRT-PCR analysis revealed the addition of 740 Y-P upregulated RUNX2, $A L P$, and $O C N$ mRNA levels in shMIR22HG hBMSCs (Fig. 6g-i).

\section{MIR22HG overexpression promoted osteogenesis by activating AKT signaling}

To further verify the relationship between MIR22HG and AKT pathway, we also investigated AKT signaling in MIR22HG overexpressed hBMSCs. Western blot results showed the level of p-AKT was significantly increased in MIR22HG overexpression group compared with NC group (Fig. 7a, b). Next, we treated MIR22HG overexpressed hBMSCs and its control group (NC) with PI3K/ AKT inhibitor LY294002. It was found that the level of pAKT was decreased when cells were treated with LY294002 (Supplementary Fig. 4C, D). As shown in Fig. $7 \mathrm{c}-\mathrm{i}$, inhibition of PI3K/AKT signaling pathway by 


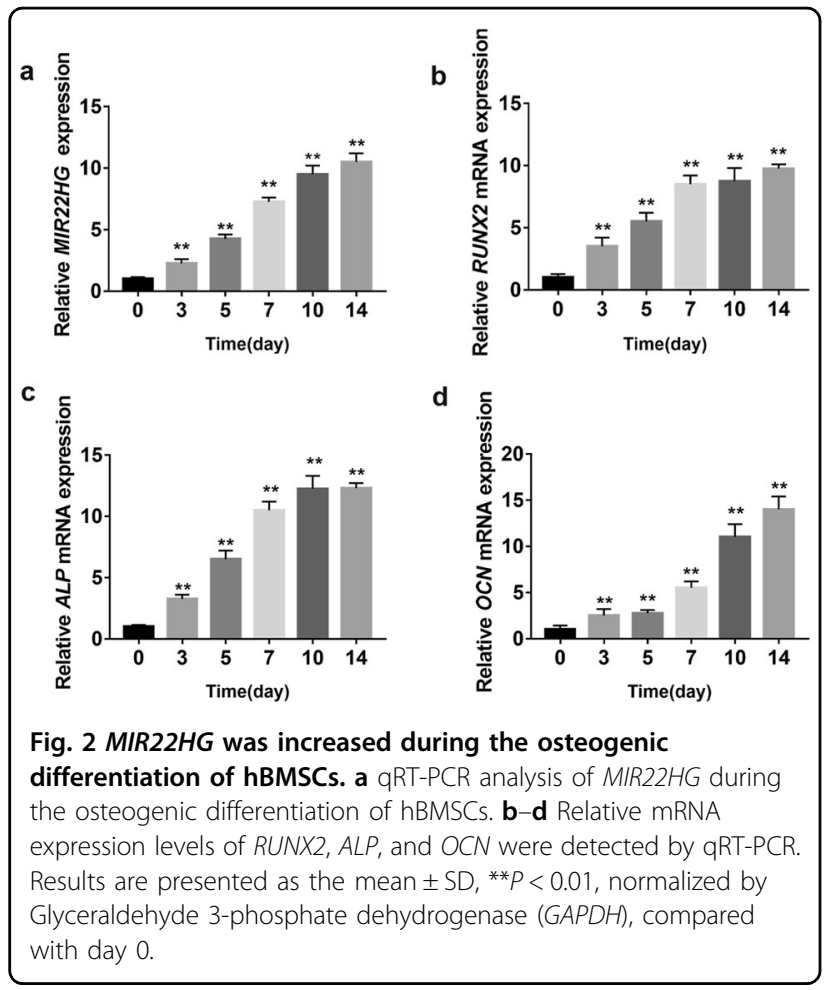

LY294002 attenuated the enhancing effect of MIR22HG overexpression on osteogenesis.

\section{The role of PTEN in MIR22HG-mediated osteogenesis}

Previous studies demonstrated that PTEN inhibits the activation of downstream proteins of AKT signaling ${ }^{23}$, we hypothesized that PTEN played a role in the MIR22HGmediated osteogenesis. Therefore, we next performed Western blot analysis of PTEN in MIR22HG knockdown or overexpressed hBMSCs. We found that MIR22HG knockdown significantly increased the level of PTEN and decreased the level of p-AKT, whereas MIR22HG overexpression had an opposite effect (Supplementary Fig. 5A, C). To determine the effects of PTEN in MIR22HGmediated osteogenesis, we transfected shMIR22HG hBMSCs and the control group (shNC) with si-PTEN. As shown in Supplementary Fig. 5B, the suppression of PTEN blocked the inhibitory effect of MIR22HG knockdown on ALP activity. In addition, PTEN overexpression plasmid and the empty control plasmid (PC) were introduced into MIR22HG overexpression hBMSCs and its control group (NC). ALP staining showed the increased ALP activity induced by MIR22HG overexpression was effectively reversed in the MIR22HG and PTEN double overexpressed hBMSCs (Supplementary Fig. 5D). These results suggested MIR22HG regulates osteogenic differentiation of hBMSCs via PTEN/AKT signaling.

\section{The distribution of MIR22HG}

Since the function of most lncRNAs is closely related to their subcellular location ${ }^{24}$, we also investigated the distribution of MIR22HG of hBMSCs by FISH assay. As shown in Supplementary Fig. 6A, MIR22HG was mainly distributed in the nucleus of hBMSCs. Consistently, subcellular fractionation assay also demonstrated that MIR22HG was primarily located in the nucleus, meanwhile the marker RNAs (MALAT1 and GAPDH) were enriched in their expected fractions (Supplementary Fig. 6B). Moreover, confocal microscopy for lncRNA MIR22HG FISH and PTEN immunostaining revealed that MIR22HG was localized predominantly in the nucleus, while PTEN was localized both in the cytoplasm and nucleus (Supplementary Fig. 6C). PTEN has been shown to play various roles in different cellular compartments. Nuclear PTEN is essential for maintaining chromosomal integrity and genomic stability. Cytoplasmic PTEN converts phosphatidylinositol-3,4,5-trisphosphate (PIP3) to phosphatidylinositol-4,5-bisphosphate (PIP2), thereby repressing PI3K/AKT pathway ${ }^{25}$. It was reported that miR-22 reduced the level of PTEN via directly combining to the 3'UTR of $\mathrm{PTEN}^{26}$. Thus, it was reasonable to hypothesize that MIR22HG regulated the level of PTEN through miR-22. As shown in Supplementary Fig. 6D, the level of miR-22 was significantly increased in MIR22HG overexpression hBMSCs. Collectively, these results indicated that MIR22HG might regulate PTEN through miR22.

\section{The role of MIR22HG in osteoclast differentiation}

It has been demonstrated that the imbalance between osteoblastic bone formation and osteoclastic bone resorption can cause osteoporosis ${ }^{27}$. Understanding the mechanisms of osteoclastogenesis is essential to develop treatments for osteoporosis. Therefore, we also investigated the role of MIR22HG in osteoclast differentiation. We found the expression level of MIR22HG was increased during RANKL-induced osteoclastogenesis of RAW264.7 cells (Supplementary Fig. 7A). Gain- and loss-of-functions studies showed MIR22HG promoted osteoclast differentiation of RAW264.7 cells (Supplementary Fig. 7B-E).

\section{Discussion}

Recent studies have reported that lncRNAs could act as key regulators of osteogenic differentiation and pathological processes of osteoporosis ${ }^{28}$. Fei et al. reported that 51 lncRNAs were differentially expressed in patients with osteoporosis compared with healthy controls ${ }^{29}$. Shen et al. showed that the expression of lncRNA HOTAIR in osteoporosis patients was significantly higher than that in normal people. They found HOTAIR suppressed osteogenic differentiation of rat BMSCs through inhibiting the Wnt $/ \beta$-catenin pathway ${ }^{30}$. Yang et al. demonstrated that 

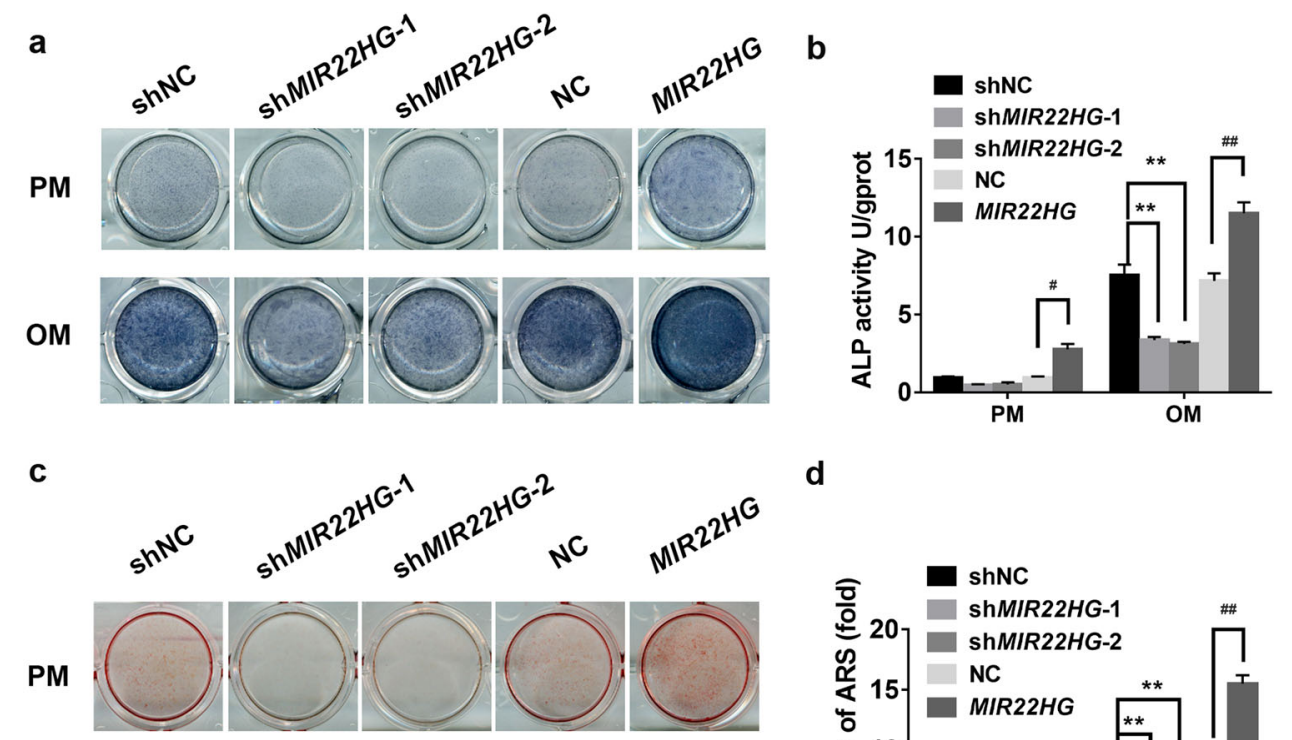

d
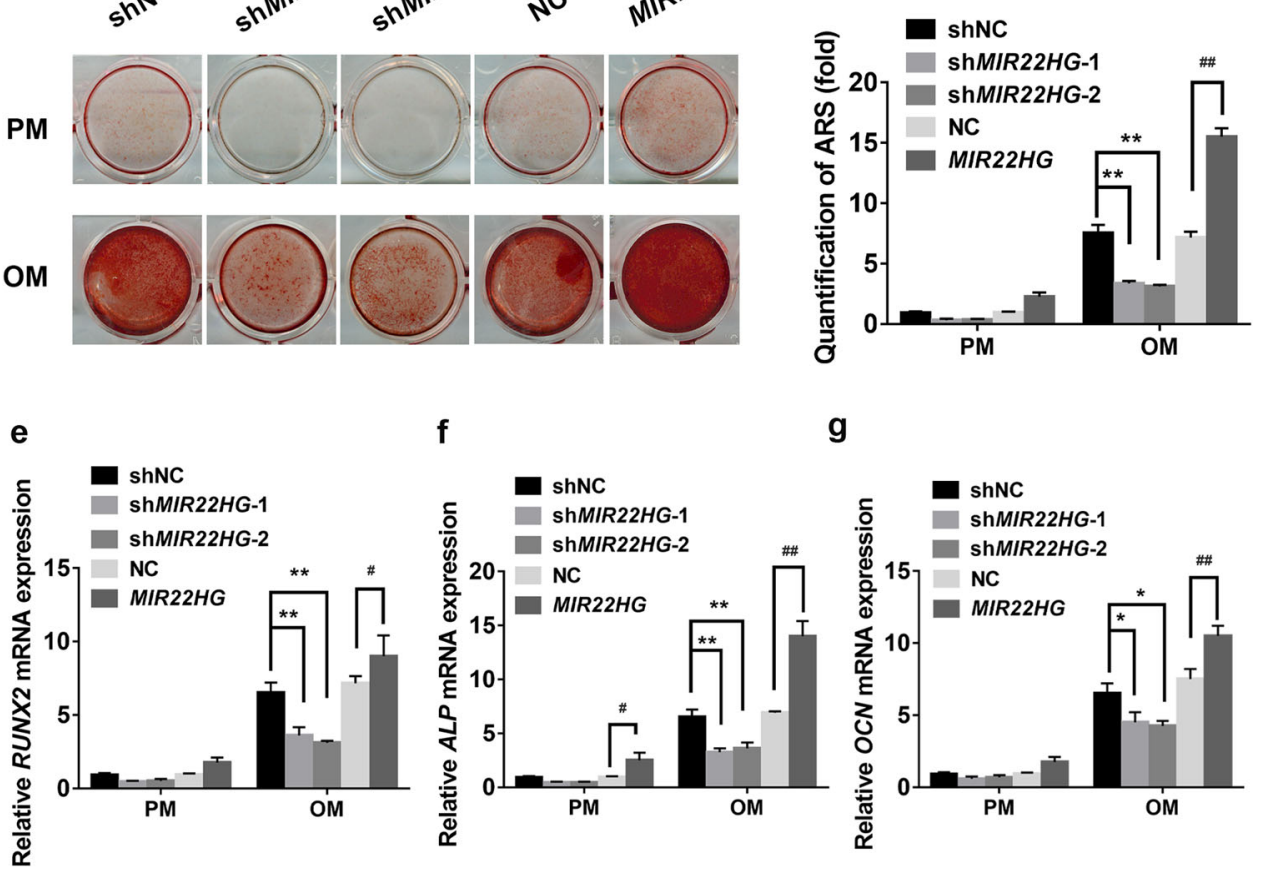

$\mathbf{g}$

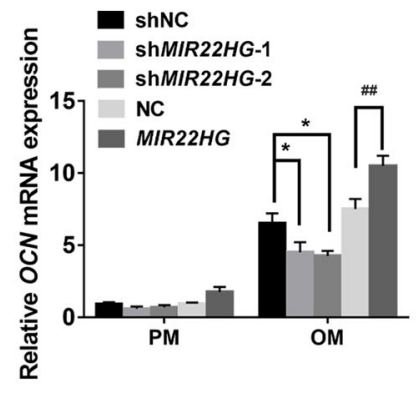

Fig. 3 The role of MIR22HG in osteogenic differentiation of hBMSCs. a Images of ALP staining in shNC, shMIR22HG-1, shMIR22HG-2, NC, MIR22HG groups. Cells were treated with proliferation medium (PM) or osteogenic medium (OM) for 7 days. b Histogram showing $7 d$ ALP activity. c, d Alizarin Red S (ARS) staining and quantification in shNC, shMIR22HG-1, shMIR22HG-2, NC, MIR22HG groups on day 14. e- $\mathbf{g}$ Relative mRNA expression levels of RUNX2, ALP, and OCN measured by qRT-PCR on day 14 of osteogenic induction. GAPDH was used for normalization. Results are presented as the mean $\pm \mathrm{SD},{ }^{*} /{ }^{\#} p<0.05,{ }^{* *} /{ }^{\# \#} p<0.01,{ }^{*}$ compared with shNC, ${ }^{*}$ compared with NC.

lncRNA ORLNC1 alleviated osteoporosis by enhancing osteogenesis through the increase of BMP2 by sponging miR-140-5 $\mathrm{p}^{31}$.

In our research, we examined the relative level of MIR22HG in mouse model of osteoporosis and found the expression of MIR22HG was lower compared with SHAM mice. We observed that MIR22HG was upregulated during the osteogenic differentiation of hBMSCs. Thus, we investigated the role of $M I R 22 H G$ in regulating osteogenic differentiation. We found MIR22HG knockdown significantly inhibited osteogenesis, while $M I R 22 H G$ overexpression enhanced osteogenesis of hBMSCs both in vitro and in vivo. These results implied the positive effect of MIR22HG in osteogenic differentiation.

Various studies have provided evidence that the PI3K/ AKT signaling plays an important role in regulating bone development. PI3K/AKT is negatively modulated by PTEN $^{32}$. The loss of PTEN function in osteoblasts leads to persistent AKT activation, and subsequently increased osteogenic differentiation $^{33}$. Previous studies discovered that several lncRNAs interacted with AKT pathway in the process of osteogenic differentiation. For example, Wu et al. showed that lncRNA HIF1A-AS2 promoted osteogenic differentiation of adipose-derived stem cells through 

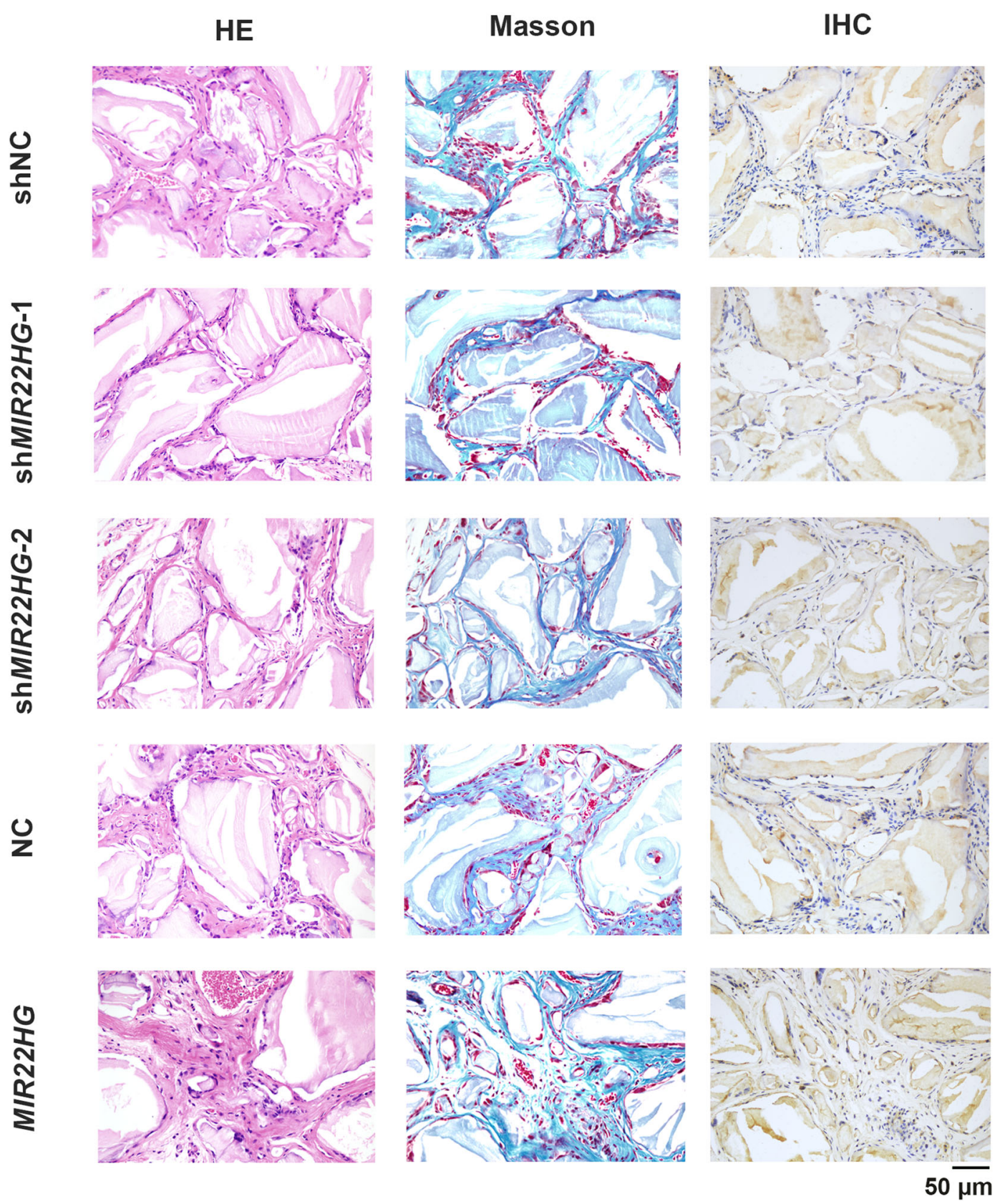

Fig. 4 MIR22HG promoted bone formation of hBMSCs in vivo. HE staining (HE), Masson's trichrome staining (Masson), and immunohistochemical staining (IHC) of osteocalcin (OCN) in shNC, shMIR22HG-1, shMIR22HG-2, NC, MIR22HG groups. Scale bar $=50 \mu \mathrm{m}$.

activating the PI3K/AKT signaling pathway ${ }^{34}$. Zhang et al. reported that AKT signaling was involved in lncRNA NKILA-mediated osteogenic differentiation in mesenchymal stem cells ${ }^{35}$.

In the current research, we found MIR22HG overexpression increased p-AKT expression, and the enhancing effect of MIR22HG overexpression on osteogenesis was abrogated by LY294002 or pcDNA3.1(+)-PTEN transfection. Moreover, MIR22HG knockdown inhibited osteogenesis of hBMSCs and downregulated the phosphorylation level of AKT. The addition of $740 \mathrm{Y}-\mathrm{P}$ or siPTEN reversed the inhibitory effect of MIR22HG knockdown on osteogenesis of hBMSCs. These results revealed that MIR22HG affected osteogenic differentiation of hBMSCs by modulating PTEN/AKT pathway.

Studies have shown that the intracellular distribution of lncRNAs is associated with their function and potential molecular roles ${ }^{36}$. Thus, we detected the subcellular 
a

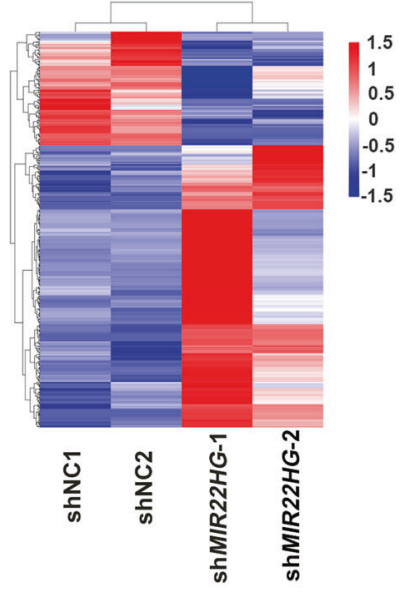

b

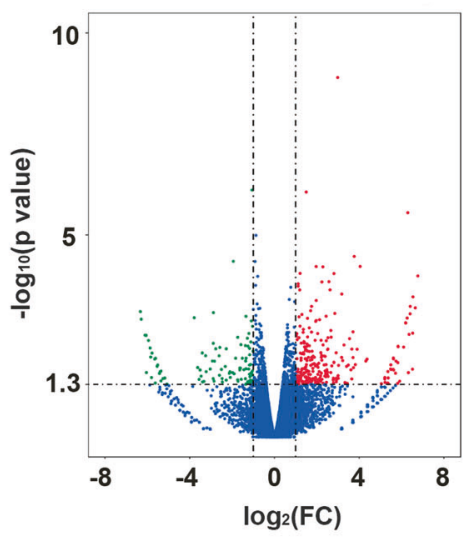

C Statistics of Downregulated Pathway Enrichment

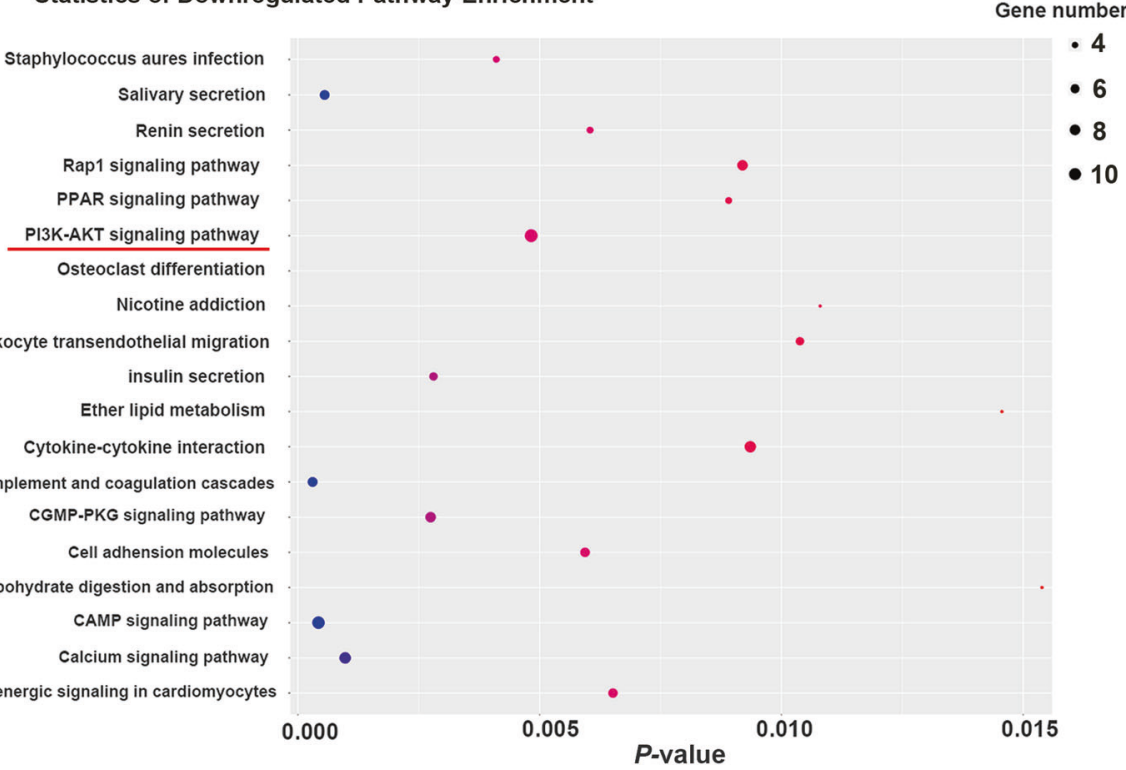

Fig. 5 Differential expression of genes between MIR22HG knockdown and shNC hBMSCs. a The differentially expressed genes in MIR22HG knockdown hBMSCs were shown in the heat map. $p$ value $<0.05$ and fold change $>2$ were set as restrictive conditions to identify the differentially expressed genes. $\mathbf{b}$ The differentially expressed genes were counted; among these genes, a total of 278 genes were upregulated and 112 genes were downregulated. c KEGG pathway analysis showed the genes downregulated by MIR22HG knockdown might be related to different pathways, among which the top-ranking enriched pathways were shown.

localization of MIR22HG by FISH and subcellular fractionation assay. The results all revealed that MIR22HG was primarily located in the nucleus of hBMSCs. However, this result was contrary to a previous study, which found that MIR22HG was mostly located in the cytoplasm of lung cancer cell lines ${ }^{12}$. This discrepancy may suggest that the role of MIR22HG varies between different cell types. To investigate the relationship between distribution of MIR22HG and PTEN/AKT pathway, we performed fluorescence colocalization microscopy analysis and observed that MIR22HG was mainly distributed in the nucleus while PTEN was presented both in the nucleus and cytoplasm. PTEN has been verified to play various roles in different cellular compartments. Multiple studies have confirmed that cytoplasmic PTEN is an antagonist of the PI3K/AKT pathway because of its lipid phosphatase activity against PIP3. Recently, growing evidence demonstrates that nuclear PTEN is important for maintaining genomic stability through critical roles in modulating DNA repair and cell-cycle arrest ${ }^{37}$. Although several components of the PI3K/AKT pathway also existed in the nucleus, such as PI3K, PIP3 and AKT, cytoplasmic but not nuclear pools of PIP3 have been discovered to be sensitive to the lipid phosphatase activity of PTEN $^{38}$. With the rapid development of highthroughput sequencing, a number of miRNAs were 


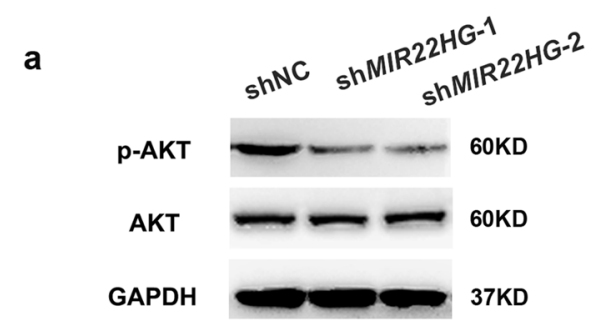

C
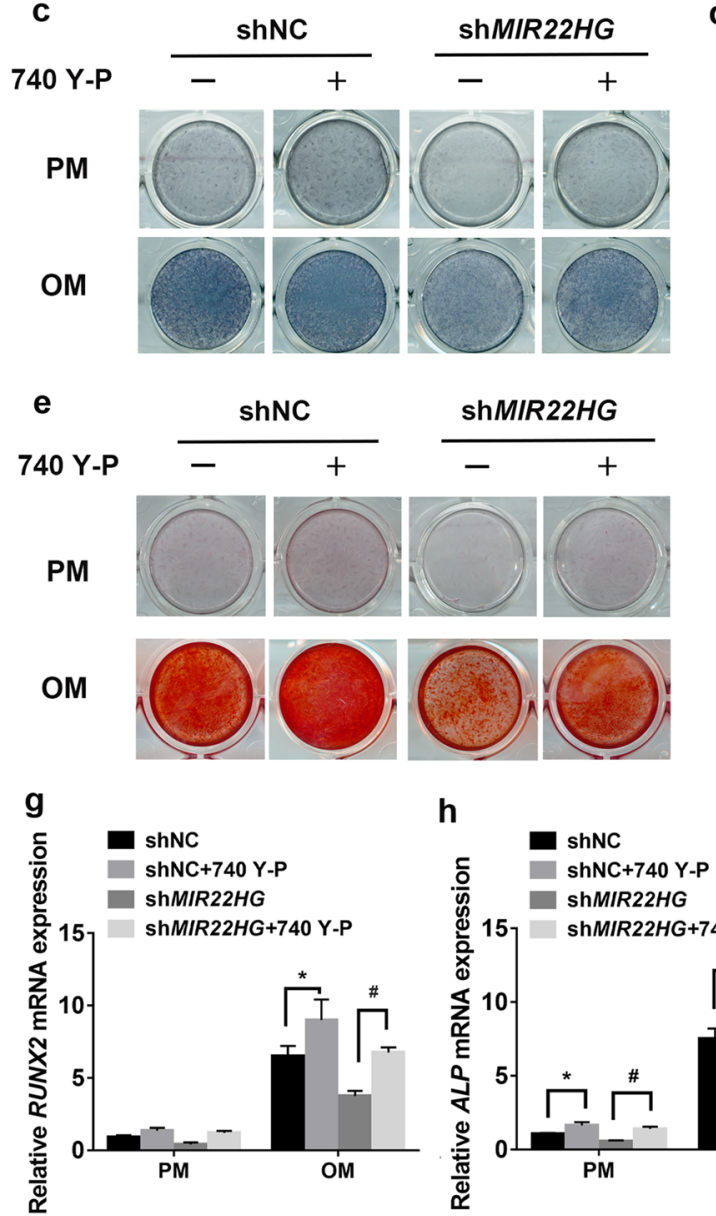

b

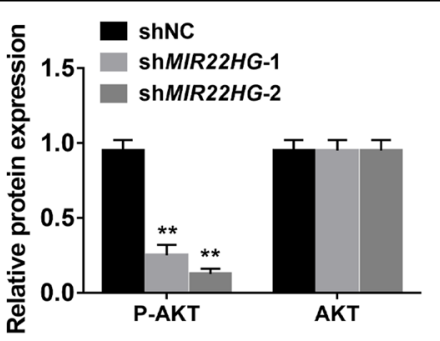

d

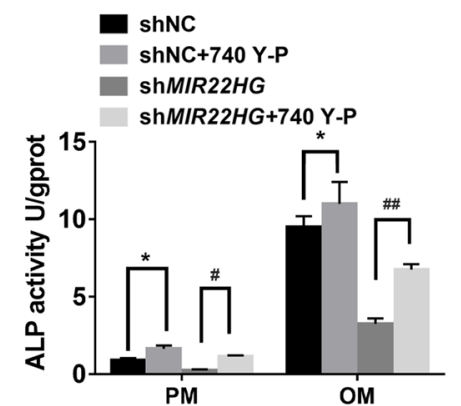

$\mathbf{f}$
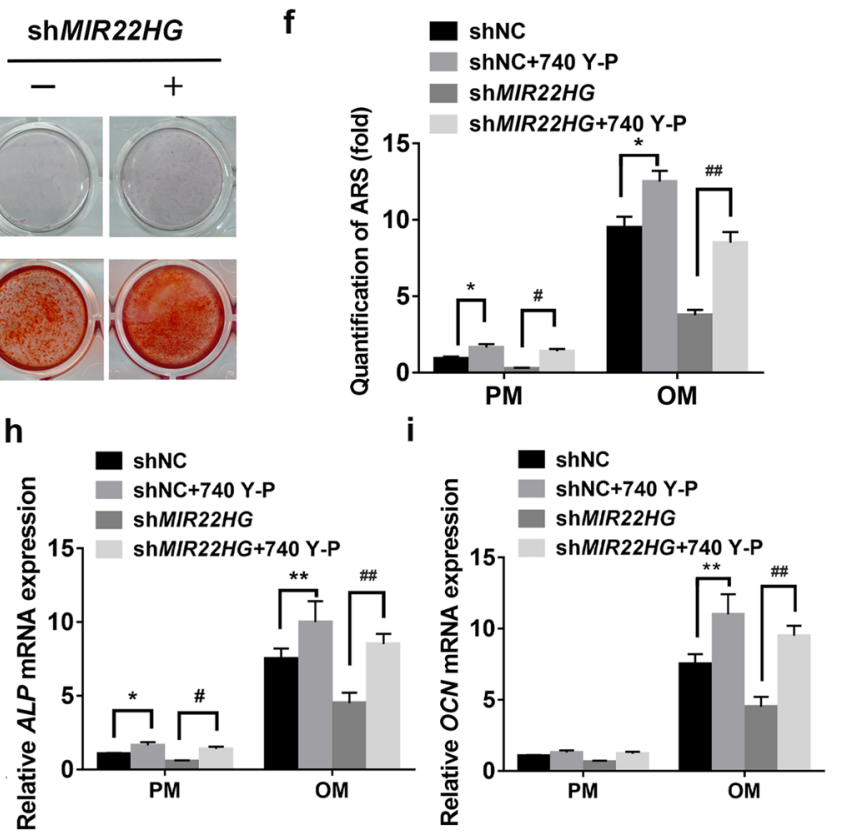

Fig. 6 MIR22HG knockdown inhibited AKT signaling. a The expression levels of total AKT and phosphorylated AKT (p-AKT) in shNC, shMIR22HG-1, and shMIR22HG-2 groups. GAPDH was used as an internal control. b The band intensities of a were analyzed by Image J software. c MIR22HG knockdown (shMIR22HG) and the control (shNC) hBMSCs were treated with proliferation or osteogenic media for 7 days. 740 Y-P (10 $\mu$ M) or DMSO (control '-') was added to the medium for 7 days and ALP staining was performed. $\mathbf{d}$ Histogram showing 7d ALP activity. e Images of Alizarin red S staining (ARS) in shNC, shMIR22HG groups treated with 740 Y-P $(10 \mu \mathrm{M})$ or DMSO (control ' - ) for 14 days. $\mathbf{f}$ Histograms showing quantification of ARS by spectrophotometry. g-i Relative mRNA expression levels of RUNX2, ALP, and OCN on day 14 after osteogenic induction. 740 Y-P (10 $\mu M$ ) was incubated for 14 days. DMSO was used as control. Results are presented as the mean $\pm \mathrm{SD},{ }^{*} /{ }^{\#} p<0.05,{ }^{* *} / \# p<0.01,{ }^{*}$ compared with shNC, ${ }^{\#}$ compared with shMIR22HG.

identified to be involved in PTEN downregulation. miR22 has been proved to downregulate PTEN in many different cell types via directly binding to the 3'UTR of $\mathrm{PTEN}^{39,40}$. In this study, we found MIR22HG overexpression significantly promoted miR-22 expression in hBMSCs. Based on these results, we speculated that MIR22HG might regulate PTEN through miR-22. More experimental studies are needed to further confirm this viewpoint.

Osteoporosis results from an imbalance between osteoblast-mediated bone formation and osteoclastmediated bone resorption. In this study, we also examined the function of MIR22HG in osteoclastogenesis and found MIR22HG promoted osteoclast differentiation of 


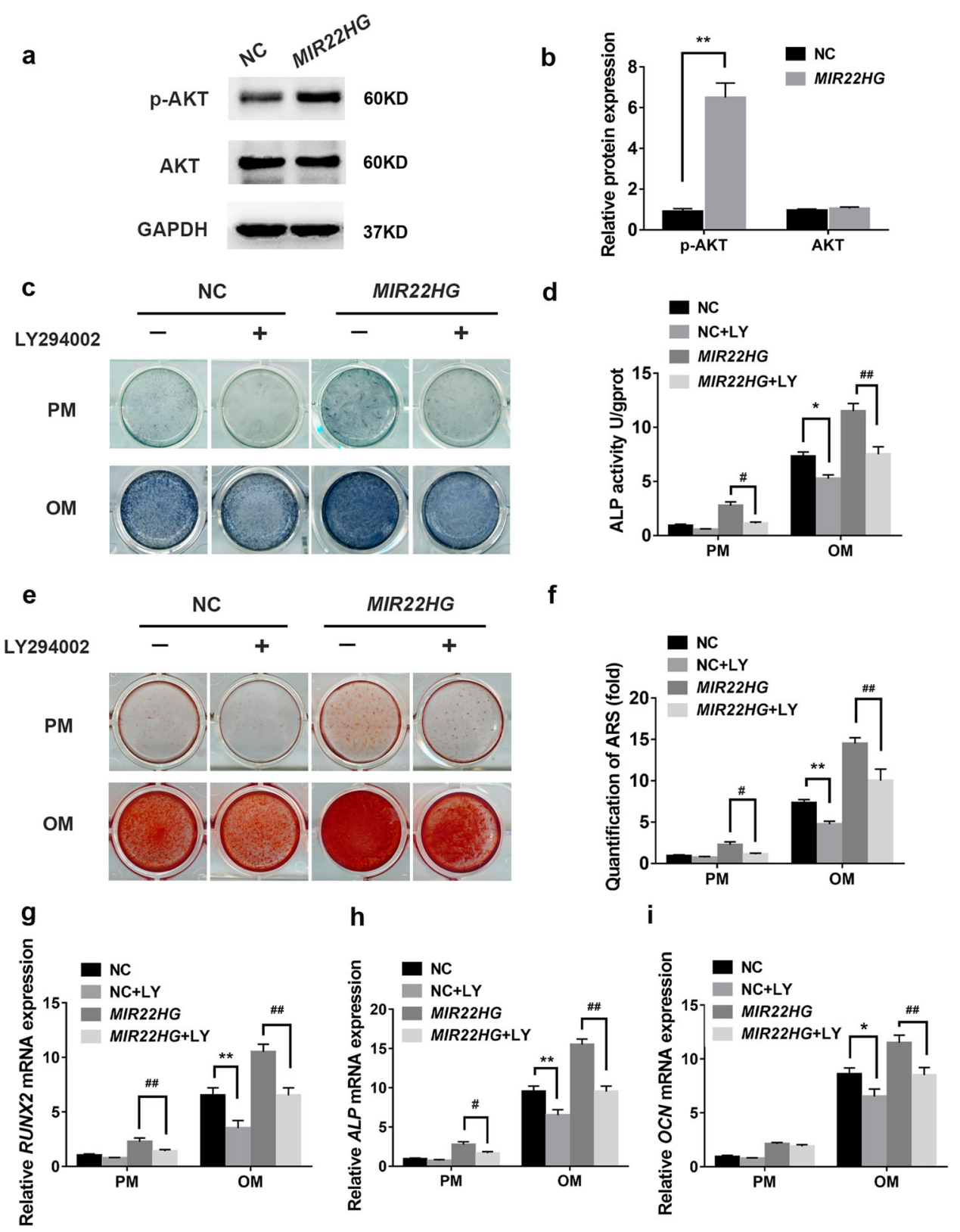

Fig. 7 MIR22HG overexpression activated AKT signaling. a The expression levels of total AKT and phosphorylated AKT (p-AKT) in NC, MIR22HG groups. GAPDH was used as an internal control. $\mathbf{b}$ The band intensities of a were analyzed by Image J software. c ALP staining in MIR22HG overexpression (MIR22HG) and the control (NC) hBMSCs with or without LY294002 (10 $\mu M$ ) treatment on day 7 of osteogenic induction. DMSO was used as control (-). d Histogram showing 7d ALP activity. e Calcium deposition in NC, MIR22HG groups treated with LY294002 (10 MM) or DMSO (control ' '-') was observed by Alizarin Red S staining on day 14 of osteogenic induction. $\mathbf{f}$ Histograms showing quantification of ARS by spectrophotometry. g-i Relative mRNA expression of RUNX2, ALP, and OCN on day 14 after osteogenic induction. LY294002 (10 $\mu M)$ was incubated for 14 days. DMSO was used as control. Results are presented as the mean $\pm \mathrm{SD},{ }^{*}{ }^{\#} p<0.05,{ }^{* *} /^{\# \#} p<0.01,{ }^{*}$ compared with NC, ${ }^{\#}$ compared with MIR22HG. LY: LY294002.

RAW264.7 cells. Sugatani et al. reported that PTEN overexpression inhibited RANKL-induced osteoclast differentiation $^{41}$. Moon et al. demonstrated that AKT stimulated osteoclast differentiation via GSK3 $\beta / N F A T C 1$ signaling cascade $^{42}$. In this study, we found that MIR22HG reduced the level of PTEN and activated AKT pathway. We reasoned that $M I R 22 H G$ might regulate osteoclastogenesis with an involvement of PTEN/AKT pathway. Future studies will try to characterize the potential interactions between MIR22HG and these pathways.

In summary, we found for the first time that lncRNA MIR22HG was downregulated in the osteoporotic model, 
and MIR22HG overexpression promoted osteogenic differentiation of hBMSCs both in vitro and in vivo. These results suggested that MIR22HG might be utilized as a novel diagnostic and therapeutic target for osteoporosis.

\section{Acknowledgements}

This study was supported by the Chinese Postdoctoral Science Foundation (2018M631442) and National Science Foundation of China (81700938\&81800942\&81772876).

\section{Author details}

${ }^{1}$ The Second Clinical Division of Peking University School and Hospital of Stomatology, 100081 Beijing, China. ${ }^{2}$ Central Laboratory, Peking University School and Hospital of Stomatology, 100081 Beijing, China. ${ }^{3}$ Department of Oral and Maxillofacial Surgery, Peking University School and Hospital of Stomatology, 100081 Beijing, China. ${ }^{4}$ Department of Orthodontics, Peking University School and Hospital of Stomatology, 100081 Beijing, China

\section{Data availability}

The authors declare that all data supporting the findings of this study are available within the paper and its supplementary information files.

\section{Conflict of interest}

The authors declare that they have no conflict of interest.

\section{Publisher's note}

Springer Nature remains neutral with regard to jurisdictional claims in published maps and institutional affiliations.

Supplementary Information accompanies this paper at (https://doi.org/ 10.1038/s41419-020-02813-2).

Received: 18 February 2020 Revised: 16 July 2020 Accepted: 16 July 2020 Published online: 30 July 2020

\section{References}

1. Sakai, T., Honzawa, S., Kaga, M., Iwasaki, Y. \& Masuyama, T. Osteoporosis pathology in people with severe motor and intellectual disability. Brain Dev. 42, 256-263 (2020).

2. Tang, C. H. Osteoporosis: from molecular mechanisms to therapies. Int. J. Mol. Sci. 21, E714 (2020).

3. Trivedi, H. D., Danford, C. J., Goyes, D. \& Bonder, A. Osteoporosis in primary biliary cholangitis: prevalence, impact and management challenges. Clin. Exp. Gastroenterol. 13, 17-24 (2020).

4. Liu, Q. et al. In vitro cell behaviors of bone mesenchymal stem cells derived from normal and postmenopausal osteoporotic rats. Int. J. Mol. Med. 41 669-678 (2018).

5. Ma, N., Tie, C., Yu, B., Zhang, W. \& Wan, J. Identifying IncRNA-miRNA-mRNA networks to investigate Alzheimer's disease pathogenesis and therapy strategy. Aging 12, 2897-2920 (2020).

6. Gao, G. C., Yang, D. W. \& Liu, W. LncRNA TERC alleviates the progression of osteoporosis by absorbing miRNA-217 to upregulate RUNX2. Eur. Rev. Med. Pharmacol. Sci. 24, 526-534 (2020).

7. Zhang, $X$. et al. Comprehensive analysis of IncRNA and miRNA expression profiles and ceRNA network construction in osteoporosis. Calcif. Tissue Int. 106 343-354 (2020).

8. Wang, J. et al. The role of IncRNAs in osteogenic differentiation of bone marrow mesenchymal stem cells. Curr. Stem Cell Res. Ther. 15, 243-249 (2020).

9. Cai, W. L. et al. LncRNA LINC00707 promotes osteogenic differentiation of hBMSCs through the Wnt/B-catenin pathway activated by LINC00707/miR145/LRP5 axis. Eur. Rev. Med. Pharmacol. Sci. 24, 18-28 (2020).

10. Wang, Z., Huang, Y. \& Tan, L. Downregulation of IncRNA DANCR promotes osteogenic differentiation of periodontal ligament stem cells. BMC Dev. Biol. 20, 2 (2020).
11. Zheng, S. et al. LncRNA MALAT1 inhibits osteogenic differentiation of mesenchymal stem cells in osteoporosis rats through MAPK signaling pathway. Eur. Rev. Med. Pharmacol. Sci. 23, 4609-4617 (2019).

12. Su, W. et al. Silencing of long noncoding RNA MIR22HG triggers cell survival/ death signaling via oncogenes YBX1, MET, and p21 in lung cancer. Cancer Res. 78, 3207-3219 (2018)

13. $\mathrm{Wu}, \mathrm{Y}$. et al. LncRNA MIR22HG inhibits growth, migration and invasion through regulating the miR-10a-5p/NCOR2 axis in hepatocellular carcinoma cells. Cancer Sci. 110, 973-984 (2019).

14. Gao, L. et al. MIR22HG as a tumor suppressive IncRNA In HCC: a comprehensive analysis integrating RT-qPCR, mRNA-Seq, and microarrays. Onco. Targets Ther. 12, 9827-9848 (2019).

15. Cui, Z., An, X., Li, J., Liu, Q. \& Liu, W. LncRNA MIR22HG negatively regulates miR$141-3 p$ to enhance DAPK1 expression and inhibits endometrial carcinoma cells proliferation. Biomed. Pharmacother. 104, 223-228 (2018).

16. Li, H. \& Wang, Y. Long noncoding RNA (IncRNA) MIR22HG suppresses gastric cancer progression through attenuating NOTCH2 signaling. Med. Sci. Monit. 25, 656-665 (2019)

17. Hu, X., Tan, Z., Yang, Y. \& Yang, P. Long non-coding RNA MIR22HG inhibits cell proliferation and migration in cholangiocarcinoma by negatively regulating the Wnt/B-catenin signaling pathway. J. Gene Med. 21, e3085-e3085 (2019).

18. Han, M. et al. Interfering with long non-coding RNA MIR22HG processing inhibits glioblastoma progression through suppression of Wnt/ $\beta$-catenin signalling. Brain 143, 512-530 (2020).

19. Jin, C. et al. RAl3 knockdown enhances osteogenic differentiation of bone marrow mesenchymal stem cells via STAT3 signaling pathway. Biochem. Biophys. Res. Commun. 524, 516-522 (2020).

20. Jin, C. et al. Inhibition of SLC7A11 by sulfasalazine enhances osteogenic differentiation of mesenchymal stem cells by modulating BMP2/4 expression and suppresses bone loss in ovariectomized mice. J. Bone Miner. Res. 32 508-521 (2017)

21. Jin, C., Wang, W., Liu, Y. \& Zhou, Y. RAl3 knockdown promotes adipogenic differentiation of human adipose-derived stem cells by decreasing $\beta$-catenin levels. Biochem. Biophys. Res. Commun. 493, 618-624 (2017).

22. Jin, C. et al. Inhibition of IncRNA MIR31HG promotes osteogenic differentiation of human adipose-derived stem cells. Stem Cells 34, 2707-2720 (2016).

23. Xi, Y., Qi, Z., Ma, J. \& Chen, Y. PTEN loss activates a functional AKT/ CXCR4 signaling axis to potentiate tumor growth and lung metastasis in human osteosarcoma cells. Clin. Exp. Metastasis 37, 173-185 (2020).

24. Luo, S. et al. Divergent IncRNAs regulate gene expression and lineage differentiation in pluripotent cells. Cell Stem Cell 18, 637-652 (2016).

25. Bononi, A. \& Pinton, P. Study of PTEN subcellular localization. Methods $\mathbf{7 7 - 7 8}$ 92-103 (2015).

26. Zhang, Y et al. MicroRNA-22 promotes renal tubulointerstitial fibrosis by targeting PTEN and suppressing autophagy in diabetic nephropathy. J. Diabetes Res. 2018, 4728645 (2018).

27. Kikuta, J. \& Ishii, M. Bone imaging: osteoclast and osteoblast dynamics. Methods Mol. Biol. 1763, 1-9 (2018).

28. Ju, C. et al. Mesenchymal stem cell-associated IncRNA in osteogenic differentiation. Biomed. Pharmacother. 115, 108912-108912 (2019).

29. Fei, Q. et al. Identification of aberrantly expressed long non-coding RNAs in postmenopausal osteoporosis. Int. J. Mol. Med. 41, 3537-3550 (2018).

30. Shen, J. J. et al. LncRNA HOTAIR inhibited osteogenic differentiation of BMSCs by regulating Wnt/ $\beta$-catenin pathway. Eur. Rev. Med. Pharmacol. Sci. 23, 7232-7246 (2019)

31. Yang, L. et al. The long non-coding RNA-ORLNC1 regulates bone mass by directing mesenchymal stem cell fate. Mol. Ther. 27, 394-410 (2019).

32. Yang, C. et al. miRNA-21 promotes osteogenesis via the PTEN/PI3K/Akt/HIF-1a pathway and enhances bone regeneration in critical size defects. Stem Cell Res. Ther. 10, 65-65 (2019).

33. Nielsen-Preiss, S. M., Silva, S. R. \& Gillette, J. M. Role of PTEN and Akt in the regulation of growth and apoptosis in human osteoblastic cells. J. Cell Biochem. 90, 964-975 (2003).

34. Wu, R. et al. Long non-coding RNA HIF1A-AS2 facilitates adipose-derived stem cells (ASCS) osteogenic differentiation through miR-665/L6 axis via PI3K/Akt signaling pathway. Stem Cell Res. Ther. 9, 348-348 (2018).

35. Zhang, Y. et al. LncRNA NKILA integrates RXFP1/AKT and NF-KB signalling to regulate osteogenesis of mesenchymal stem cells. J. Cell Mol. Med. 24, $521-529(2020)$

36. Batista, P. J. \& Chang, H. Y. Long noncoding RNAs: cellular address codes in development and disease. Cell 152, 1298-1307 (2013). 
37. Dai, W. Unmasking PTEN's nuclear functions. Cell Cycle 15, 3341-3342 (2016).

38. Lindsay, $Y$. et al. Localization of agonist-sensitive Ptdlns $(3,4,5)$ P3 reveals a nuclear pool that is insensitive to PTEN expression. J. Cell Sci. 119, 5160-5168 (2006).

39. Wang, X., Wang, Y., Kong, M. \& Yang, J. MiR-22-3p suppresses sepsisinduced acute kidney injury by targeting PTEN. Biosci. Rep. 40 BSR20200527 (2020).
40. Wang, L. et al. The microRNA miR-22 represses Th17 cell pathogenicity by targeting PTEN-regulated pathways. Immunohorizons 4, 308-318 (2020).

41. Sugatani, T., Alvarez, U. \& Hruska, K. A. PTEN regulates RANKL- and osteopontin-stimulated signal transduction during osteoclast differentiation and cell motility. J. Biol. Chem. 278, 5001-5008 (2003).

42. Moon, J. B. et al. Akt induces osteoclast differentiation through regulating the GSK3ß/NFATc1 signaling cascade. J. Immunol. 188, 163-169 (2012). 